\title{
Dual Use of Veterans Health Administration and Indian Health Service: Healthcare Provider and Patient Perspectives
}

\author{
B. Josea Kramer, PhD ${ }^{1,2}$, Rebecca L. Vivrette, $M A^{7}$, Delight E. Satter, $M P H^{3}$, \\ Stella Jouldjian, MSW, MPH', and Leander Russell McDonald, $\mathrm{PhD}^{4}$
}

'Geriatric Research Education and Clinical Center, VA Greater Los Angeles Healthcare System, Sepulveda, CA, USA; ${ }^{2}$ David Geffen School of Medicine, University of California at Los Angeles, Los Angeles, USA; ${ }^{3}$ Center for Healthcare Policy and Research, University of California at Los Angeles, Los Angeles, CA 90024, USA; ${ }^{4}$ Cankdeska Cikana Community College, P.O. Box 269, Fort Totten, ND 58335, USA.

BACKGROUND: Many American Indian and Alaska Native veterans are eligible for healthcare from Veterans Health Administration (VHA) and from Indian Health Service (IHS). These organizations executed a Memorandum of Understanding in 2003 to share resources, but little was known about how they collaborated to deliver healthcare.

OBJECTIVE: To describe dual use from the stakeholders' perspectives, including incentives that encourage cross-use, which organization's primary care is "primary," and the potential problems and opportunities for care coordination across VHA and IHS.

PARTICIPANTS: VHA healthcare staff, IHS healthcare staff and American Indian and Alaska Native veterans.

APPROACH: Focus groups were conducted using a semi-structured guide. A software-assisted text analysis was performed using grounded theory to develop analytic categories.

MAIN RESULTS: Dual use was driven by variation in institutional resources, leading patients to actively manage health-seeking behaviors and IHS providers to make ad hoc recommendations for veterans to seek care at VHA. IHS was the "primary" primary care for dual users. There was little coordination between VHA and IHS resulting in delays and treatment conflicts, but all stakeholder groups welcomed future collaboration.

CONCLUSIONS: Fostering closer alignment between VHA and IHS would reduce care fragmentation and improve accountability for patient care.

KEY WORDS: veterans; rural health; qualitative research; patient preferences; health services research.

J Gen Intern Med 24(6):758-64

DOI: $10.1007 / \mathrm{s} 1$ 1606-009-0962-4

(C) Society of General Internal Medicine 2009

Received September 15, 2008

Revised January 22, 2009

Accepted March 12, 2009

Published online April 18, 2009

\section{INTRODUCTION}

The Veterans Health Administration (VHA) and the Indian Health Service (IHS) represent two independent branches of the federal health care system. In 2003, these organizations executed a Memorandum of Understanding (MOU) to share resources and improve healthcare access for American Indian and Alaska Native (AIAN) populations. ${ }^{1}$ With the goal of optimizing health outcomes, the MOU encourages local collaborations without addressing coordination of care across these two systems with significant organizational differences involving eligibility, benefits, and resources.

VHA, a branch of the Department of Veterans Affairs, provides comprehensive, free or low-cost healthcare to eligible veterans with variable co-payments based on need, serviceconnected injuries or illnesses (SCI) and income. ${ }^{2}$ IHS, an agency within the Department of Health and Human Services, has primary responsibility for patient care and public health services to enrolled members of federally recognized tribes and their descendants. IHS facilities can be administered either as part of the federally administered direct service program or as tribally administered health programs. VHA services are available in all 50 US states, and include the broad range of expertise and programs that are typical of urban, hospitalbased programs. In contrast, IHS facilities are present in 35 states, primarily on or near rural reservations, and focus on providing primary health care at no cost to patients, with limited specialty care and inpatient services. VHA has a standard benefits package and allocates priority status for limited VHA resources (e.g., dental care or admission to a skilled nursing home) based on the extent to which SCI contribute to medical conditions. IHS has no standard benefits package, ${ }^{3}$ and care is allocated on the basis of patients' health status. $^{4}$

There are important differences in funding for VHA and IHS. While VHA funding allocation is based on actual workload, the IHS allocation is based on population per capita, and is insufficient to meet the total healthcare needs of the eligible AIAN people, including Contract Health Services (CHS) by nonIHS providers, such as specialty care. ${ }^{3,5}$ The full range of IHS services may only be available at a home reservation facility, and therefore, depending on the location, some IHS enrollees will not be eligible for IHS-CHS. ${ }^{6}$

Use of multiple healthcare systems is both permitted and common among $\mathrm{VHA}^{7-12}$ and $\mathrm{IHS}^{13-16}$ enrollees, despite the potential for lower quality when no organization is primarily accountable for care. ${ }^{17-18}$ Approximately one-quarter of IHS- 
enrolled veterans use VHA for health care; commonly receiving treatment for diabetes mellitus, hypertension or cardiovascular disease in both organizations. ${ }^{19}$ In the few reports of VHAIHS dual use, veterans' preference is variously attributed to IHS care in general ${ }^{20}$ or to VHA for specific types of care based on quality, cost and customer service. ${ }^{21}$

To further understand dual utilization patterns, we conducted focus group research to explore organizational and behavioral factors that affect VHA-IHS dual use, including 1) What factors drive dual use? 2) Which primary care is "primary?" 3) How is care coordinated? and 4) Is there interest in collaboration between VHA and IHS by healthcare field staff?

\section{METHODS}

Focus group interviews were conducted with three stakeholder categories: AIAN veterans, VHA staff and IHS staff. Our aim was to systematically describe dual use and report the range of experiences and opinions by triangulating responses from each stakeholder category to address each study question. The sampling frame was constructed by identifying pairs of VHA and reservation-based IHS facilities with overlapping catchment areas and a minimum of 70 dual users (determined by linked and merged administrative data ${ }^{19}$ ). As the IHS facilities were located on reservations, their location determined the tribal community. With the advice of our advisory committee, we identified clusters of VHA-IHS-tribal communities to represent variation in geographic and indigenous culture areas, IHS governance (i.e., federal direct or tribal) and distance between VHA and IHS. All IHS facilities were located in health profession shortage areas. We sought to recruit 8-12 individuals at each site. We invited 68 healthcare providers to participate via mail and telephone based on identification by their respective chiefs of staff (COS) as being knowledgeable regarding provision of services to AIAN veterans. The participation rate was $81 \%(n=55)$ across a range of disciplines. Recruitment of veterans was performed independently in tribal communities to respect tribal sovereignty. Local leadership (e.g., veteran liaison to tribal council) identified veterans and invited their participation verbally and/or using a project invitation letter, with a total of 38 AIAN veterans agreeing to participate.

Trained focus group moderators guided 90-minute focus group interviews using a semi-structured discussion guide. Audio recordings of the sessions were transcribed verbatim. Qualitative software ${ }^{22}$ was used for coding, analysis and text retrieval. Interpretation followed grounded theory in which conceptual themes were derived from participants' statements, using a constant comparative method. ${ }^{23-24}$ The data collection team identified general themes that emerged in each focus group immediately after each interview and the analysis team delineated, refined and tested a data coding schema. Data coding was performed independently by three coders; intercoder reliability was tested on approximately one-fourth of the coding structure across all transcripts by revolving pairs of coders. In the rare case of coding discrepancy, a three-person consensus process resolved conflicts. All coded statements were reviewed for accuracy, and if technical errors were discovered, codes were re-assigned before analysis. The study was approved by the Institutional Review Boards (IRB) of local tribal governments where interviews took place, as well as the VA Greater Los Angeles Healthcare System, IHS, and the University of California at Los Angeles.

\section{RESULTS}

Table 1 indicates the characteristics of the VHA-IHS-tribal community clusters and Table 2 summarizes participants' characteristics. We were unsuccessful in recruiting participation from an IHS facility in Cluster D, and the associated AIAN population was recruited from a neighboring off-reservation inter-tribal community. These veterans had experience seeking care at the VHA and IHS facilities in the cluster and their responses confirmed that data saturation ${ }^{25-26}$ had been achieved. Table 3 summarizes consistency in spontaneous responses 1) across all stakeholder categories regarding experiences of receiving or delivering healthcare, and 2) beliefs by healthcare providers regarding organizational barriers and opportunities to improve coordination of care for mutual patients.

Table 1. Characteristics of Clustered Healthcare Facilities and American Indian and Alaska Native (AIAN) Communities

\begin{tabular}{|c|c|c|c|c|c|c|c|c|c|c|c|}
\hline \multirow{3}{*}{$\begin{array}{c}\text { Characteristics } \\
\text { VHA-IHS-Tribal community clusters }\end{array}$} & \multicolumn{4}{|c|}{$\begin{array}{c}\text { Veterans Health } \\
\text { Administration Facility }\end{array}$} & \multicolumn{3}{|c|}{$\begin{array}{l}\text { Indian Health } \\
\text { System Facility }\end{array}$} & \multicolumn{4}{|c|}{ AIAN veteran community } \\
\hline & \multirow[b]{2}{*}{ A } & \multirow{2}{*}{ B } & \multirow{3}{*}{ c } & \multirow{3}{*}{$\frac{D}{-}$} & \multicolumn{2}{|c|}{ Tribal } & \multirow{2}{*}{$\frac{\text { Federal }}{\text { B }}$} & \multicolumn{3}{|c|}{ Reservation } & \multirow{2}{*}{$\frac{\text { Non-Reservation }}{D}$} \\
\hline & & & & & A & C & & A & B & C & \\
\hline \multicolumn{10}{|c|}{ Geographic areas (distance between facilities') } & & \\
\hline Southern Plains (83 mi.) & $\boldsymbol{\nu}$ & & & & $\boldsymbol{r}$ & & & $\prec$ & & & \\
\hline Southwest (179 mi.) & & $\boldsymbol{r}$ & & & & & $\mathscr{r}$ & & $\boldsymbol{r}$ & & \\
\hline Northwest (29 mi.) & & & $\swarrow$ & & & $\prec$ & & & & $\prec$ & \\
\hline $\begin{array}{l}\text { Northern Plains (133 mi.) } \\
\text { Facility type }\end{array}$ & & & & $\prec$ & & & & & & & $\prec$ \\
\hline Inpatient \& Outpatient & $\boldsymbol{r}$ & $\boldsymbol{r}$ & 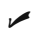 & $\mathscr{}$ & $\mathscr{\swarrow}$ & & $\mathscr{}$ & & & & \\
\hline Outpatient only & & & & & & $\swarrow$ & & & & & \\
\hline Located in rural county ${ }^{2}$ & & & & 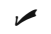 & 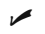 & & 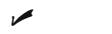 & $\swarrow$ & 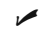 & & $\swarrow$ \\
\hline
\end{tabular}

${ }^{1}$ Distance calculated using zip code centroids between VHA and IHS facilities

${ }^{2}$ Source, Rural Assistance Center, http://ims2.missouri.edu/rac/amirural/, accessed 2/19/09 
Table 2. Characteristics of Focus Group Participants by Stakeholder Group

\begin{tabular}{ll}
\hline \hline Stakeholder group and characteristics & Number or proportion \\
\hline AIAN veterans & $\mathrm{n}=38$ \\
Sex - male & $89.4 \%$ \\
Mean age & 61.1 years \\
Period of military service & \\
WWII & $7.9 \%(3)$ \\
Korean War & $10.5 \%(4)$ \\
Vietnam & $65.8 \%(25)$ \\
Persian Gulf & $10.5 \%(4)$ \\
Other & $18.4 \%(7)$ \\
VHA Healthcare Providers & $\mathrm{n}=25$ \\
Professional discipline & \\
Associated Health Professions & $60.0 \%(15)$ \\
Administration & $28.0 \%(7)$ \\
Medicine & $12.0 \%(3)$ \\
IHS Healthcare Providers & $\mathrm{n}=30$ \\
Professional discipline & \\
Associated Health Professions & $53.3 \%(16)$ \\
Administration & $26.6 \%(8)$ \\
Medicine & $20.0 \%(6)$ \\
\hline
\end{tabular}

\section{What Factors Drive Dual Use?}

Patient Factors: Veterans' Perspectives. Patients actively matched healthcare resources to their medical needs, generally using IHS for primary care and VHA for specialty care. VHA was an important source of care that could not be obtained elsewhere. For instance, veterans chose VHA to supplement IHS primary care if they were unable to obtain private health insurance or were ineligible for IHS-CHS. Some veterans used VHA emergency rooms to access medical care more quickly than might have been possible if they were solely reliant on IHS-CHS.
Some veterans' decisions about where to access care were based on saving money for the healthcare organization and the community that it represents.

Veteran: We had a limited amount of money in Indian healthcare and, so if it is something expensive, I'd go to the VA.

Veteran: The reason [that] I am kind of reluctant to go to the VA [is because] I'm non-combat...When ...I seen some of the Iraqi veterans, just kids, missing limbs and psych problems, and guys like, Vietnam vets... These guys need it more than me.

Organizational Factors: VHA and IHS Providers' Perspectives. VHA providers generally perceived AIAN veterans as taking an active role in determining which healthcare organization offered the best resources at the lowest cost for any particular medical need. VHA was used mainly for limited and specific services.

VHA: A lot of patients will come to the VA for conditions for which they're service-connected because then they pay no visit co-payment, they pay no medication co-payment and then they may go to IHS for everything else.

Clinicians recognized that they might not be able to provide all the care that any AIAN veteran may require if services were limited by SCI rating.

VHA: If we can't get a veteran in, if the rating is not high enough...they may choose to go to the IHS ...[for] care that they can get there...like dental.

Table 3. Agreement across Focus Groups on Key Issues about Dual Use and Coordination Between VHA and IHS by Stakeholder Category

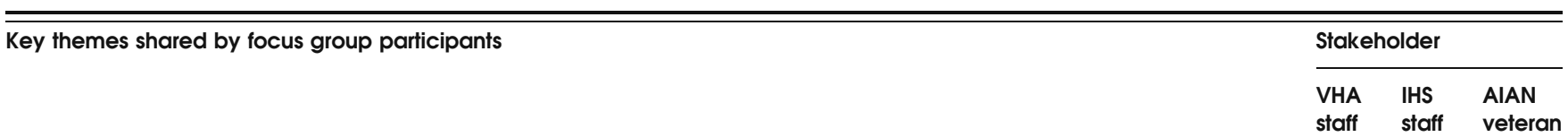

What drives dual use?

Patients access IHS for primary care, VHA for specialty care

Patients access care at VHA for service-connected injury/illness (lower cost)

IHS refers patients to VHA when services are unavailable or are costly to contract

Patients conserve resources for organization/community by accessing the other system

Patients match specific healthcare needs to organization with resources at the lowest cost

Which primary care is "primary" for dual users?

IHS is the "primary" primary care for dual users

Patient is asked to choose which organization is primary

How is care coordinated between VHA and HIS?

Lack of systematic primary care coordination between organizations

Informal, ad hoc communications between clinicians

Patients actively manage their own care across systems

Referrals are unidirectional (IHS refers to VHA)

Lack of systematic communication between organizations about medical screening and assessment, treatment regimens

and discharge planning

Lack of knowledge about other organization's eligibility structure and benefits reduces appropriate referrals

Patient assessments and laboratory tests are conducted independently (i.e., may be duplicated)

Is there interest in fostering closer collaboration between VHA and HIS?

Improve communication between providers about medical records and services received

Shared Electronic Health Record (EHR)

Identify a point of contact at other organization to improve coordination between providers

Improve knowledge about other organization's resources, benefits structure and eligibility

Provide joint training, share continuing education costs

$\begin{array}{lll}\mathrm{x} & \mathrm{x} & \mathrm{x} \\ \mathrm{x} & \mathrm{x} & \mathrm{x} \\ \mathrm{x} & \mathrm{x} & \mathrm{x} \\ \mathrm{x} & & \mathrm{x} \\ \mathrm{x} & & \mathrm{x} \\ \mathrm{x} & \mathrm{x} & \mathrm{x} \\ \mathrm{x} & \mathrm{x} & \\ \mathrm{x} & \mathrm{x} & \mathrm{x} \\ \mathrm{x} & \mathrm{x} & \mathrm{x} \\ \mathrm{x} & \mathrm{x} & \mathrm{x} \\ \mathrm{x} & \mathrm{x} & \mathrm{x} \\ \mathrm{x} & \mathrm{x} & \\ & & \\ \mathrm{x} & \mathrm{x} & \\ \mathrm{x} & \mathrm{x} & \\ \mathrm{x} & \mathrm{x} & \mathrm{x} \\ \mathrm{x} & \mathrm{x} & \mathrm{x} \\ \mathrm{x} & \mathrm{x} & \mathrm{x} \\ \mathrm{x} & \mathrm{x} & \\ \mathrm{x} & \mathrm{x} & \\ & & \end{array}$


IHS healthcare providers actively "referred" (IHS providers described recommendations as "referrals") patients who were known to be veterans to VHA for specialized services that were not available locally or would be costly through IHS-CHS. These referrals might be facilitated, in part, by IHS benefits counselors. Typically, referrals were for imaging, subspecialty care and extensive or complicated surgical procedures. Other benefits included VHA's wider range of free or low cost medications. From their perspective, these providers were practicing good medicine and effective cost containment both for the patient and for the healthcare institution.

IHS: [There are] more patients than contract dollars to pay for [their medical needs that exceed local resources] and if there's anyway to get a CT scan without [the procedure] coming out of [the patient's] pocket, [I] try and get them in at the VA.

While some veterans appreciated these referrals, opining that VHA offered first-rate care at some facilities based on their experiences of quality, courtesy and well-organized clinic schedules, others described their frustration at the lack of local IHS expertise.

Veteran: [When IHS providers] don't know what your problem is [they say] 'we're going to have to send you somewhere.' Then they ask you, 'are you a veteran?' And then you say yes. 'Well, you'll have to go to the VA to have yourself checked out because we don't know what you've got.'

While there were no personal experiences, providers and patients reported that redirection of patients to VHA from public and private medical facilities had occurred in clusters A, B and D. All stakeholder groups voiced concerns that there may be financial disincentives for VHA to accept additional patients, rather than effectively outreach to Native veterans.

\section{Which Primary Care Is "Primary" for Dual Users?}

Both organizations enrolled patients for primary care and required patients to have primary care assessments in order to access subspecialty consults or to receive diagnostic tests or procedures. Nevertheless, VHA clinicians appeared to recognize IHS as the usual provider. Dual users did not make the distinction that primary care was intended to manage all types of medical care. When asked which care is "primary," the following was a typical response:

Veteran: Well, as far as I'm concerned... it's shared. Specialty VA. You got everyday care, IHS. Because it's too far for me to go to the VA.

Clinicians recognized that dual use was an AIAN veterans' right and a challenge to managed care practices.

VHA: I think it's important to remember, I know it's a difficult situation, but an IHS veteran has the right to use either system and both systems if they wish. And it makes it difficult for providers.

Clinicians in both organizations discussed treatment conflicts, particularly for patients with diabetes mellitus, as a common occurrence with dual use. With patient safety in mind, some clinicians asked AIAN veterans to choose a single primary care provider. Most often, patients were given information and the burden of resolving treatment conflicts.

VHA: I usually would explain to them the dangers related to dual care and basically ask them to choose who they wanted to manage their primary care at this time, because it didn't work with one provider adjusting medications, me adjusting medications, it was dangerous to them.

\section{How Is Care Coordinated Between VHA and IHS?}

Generally, healthcare was not coordinated for mutual patients. Although both organizations could share information through medical releases, veterans were dissatisfied with the burdensome process when it was made available as an option. Since medical information was not routinely shared, treating chronic health conditions was challenging, especially when providers were unaware of their counterpart's recommendations of treatments, including medications and dosage.

VHA: The frustration I felt as a primary care provider... [with] dual-care [is] the Native American [veteran] getting care at their local clinic and then coming here and not really knowing what medications were changed and what medications they were on and then also running primary care through the VA, and there was not a good way to communicate between them, the two clinics.

IHS: There may be a great reason for that [VHA clinician] deciding they need to be on a [certain] medication rather than another but you don't know that because the patient can't communicate that or doesn't or something. So, then ...you ... have a conflict...

Lack of primary care coordination resulted in longer patient visits in primary care settings to identify potential conflicts in treatment. Clinicians in both organizations voiced frustrations about instances of duplicated care and of delays in treatment since both primary care physicians must independently review a patient's symptoms and disease process.

There was also little formal coordination for other types of healthcare. For the most part, coordination between providers occurred on an ad hoc basis, with clinicians informally contacting counterparts or key contacts that had been cultivated in the opposite system. Unlike healthcare organizations paid by IHS-CHS, VHA did not respond to IHS "referrals" with follow-up information on test results, consultation recommendations or discharge plans. For patients, information-sharing experiences varied since many patients who actively managed their own care also facilitated communication across systems. In some cases, patients hand-carried their own records between providers; others shared recommendations for care jotted on a prescription pad; others reported that their doctors often talked to each other by telephone.

Mostly, referrals for specialized types of services were unidirectional. VHA providers rarely referred patients to IHS. Unless there was a specific agreement between VHA and IHS facilities to co-manage patients (e.g., referral for specialized 
services) or resources (e.g., pharmaceutical dispensing), there was no formal coordination of care for mutual patients.

Successful coordination was also impeded by healthcare providers' lack of awareness of the other system's enrollment and eligibility policies, benefits structure or healthcare resources. For instance, since a veteran's enrollment status and eligibility rating would be unknown to IHS, a patient may be referred to VHA but be ineligible for care. This situation was compounded by differences in the definition of "eligible veteran" from the VA handbook and the more generous community definition of any military experience. IHS personnel were generally unaware of the eligibility criteria and services at VHA. As a result, their expectation of gratis care for veterans at VHA may not be met, leaving patients, IHS facilities and/or tribes with unexpected costly bills for medical care. In some cases, where veterans were transferred to VHA for emergent care, eligibility for VHA air or ambulance transportation from a distant rural area may not be determined until after the episode of care. Some IHS clinicians also wrongly assumed that their standard benefits, such as dental care, were also readily available to all-comers at VHA. In a similar manner, some VHA staff may not appreciate the differences in organizational structure and eligibility of patients for direct federal and tribal health programs, leading to false assumptions that all AIAN veterans were eligible for the same types of care.

\section{Is There Interest in Fostering Closer Collaboration Between VHA and IHS?}

In each focus group, healthcare staff spontaneously recommended ways to foster closer collaboration between VHA and IHS to resolve obstacles in the seamless transition of care. A key issue was improving communication about medical records, including previous work-ups and hospital discharges, especially when VHA served a consulting role or provided specialty or inpatient services in an episode of care. One recommendation was to routinely share information through an "opt out" release of medical records, rather than requiring a separate release of medical information for each visit.

A single electronic health record (EHR) was the favored solution to improve coordination, expedite referrals and reduce duplication. The technology of the EHR might also allow VHA and IHS to jointly meet quality indicators. Unintended overlaps could be avoided as part of the natural process of care. In addition to improving care and reducing duplication, sharing information was perceived as a cost-saving measure to the federal government, for instance when adhering to American Diabetes Association (ADA) clinical guidelines. Another solution was to adopt a direct referral model between IHS clinics and VHA to extend healthcare resources to AIAN veterans' living in rural areas.

IHS: If I'm taking care of [a veteran] who goes to the VA and says he's diabetic and does his eyecare and dental care there, and I access the record to see. He won't have to be seen in the eye clinic and dental clinic here because he's got that ADA requirement for the year, and I have access to see he's stable and [the annual evaluation] is okay, and I don't have to duplicate it. So, that would be nice to be able to share in that information through the EHR.
Another key to closer collaboration was improving knowledge of each other's organizations. Appropriate referrals to VHA from the IHS would be a significant step toward resource sharing that would benefit both organizations financially.

VHA: That's part of ... co-managed care ... to have IHS successfully refer [AIAN] to us, to VA, for specialty care... And that's a win/win situation because then IHS is not paying for the high-end specialty care; we know that we're getting patients that have been screened appropriately by IHS...

Education about local resources and eligibility in each system was important to both VHA and IHS participants. Identifying points of contact was suggested to facilitate healthcare processes for mutual patients. Awareness of local resources was seen as a first step in identifying how gaps in the continuum of care might be filled. One example suggested was that while VHA may not have home care or hospice resources in distant rural areas, IHS or tribal programs may have local services in place. Sharing the costs of continuing education and staff development programs was another recommendation to be fiscally responsible while increasing the opportunities for healthcare professionals to interact and network.

Veterans and healthcare staff envisioned different methods to shape and support closer alignment between VHA and IHS on behalf of AIAN veterans and communities. Some veterans saw improved coordination as a political issue that would require political action by tribal governments, Congress and advocacy groups.

Veteran: I think to get any changes to be made, you're going to have to get the real attention of the tribal elected officials [and] the national organizations such as the National Indian Health Board [and] Congress to make the changes.

Healthcare providers believed the mechanism was already in place but underutilized. They perceived the MOU as enabling new opportunities between local/regional VHA and IHS/tribal facilities to improve quality of care and access. In addition, providers described the MOU as a foundation for organizational change by developing a more integrated healthcare delivery system for AIAN veterans through systematic sharing of information, resources and expertise.

\section{DISCUSSION}

By triangulating the experiences, attitudes and beliefs of these three stakeholder groups, we were able to systematically describe the drivers of dual use and address which organization delivers "primary" primary care. ${ }^{27}$ Dual use resulted from the behaviors of healthcare providers, as well as patients, in response to healthcare resource shortages at IHS/tribe facilities. An ad hoc system has developed to extend IHS/tribe rural health networks to include VHA urban partners for diagnostic, specialty and hospital-based care for veterans. The lack of formalized relationships to clearly define $\operatorname{roles}^{28}$ and support communication on mutual patients across organizations and rural/urban settings has resulted in potential overlaps and 
treatment conflicts. From the clinicians' perspective, these conflicts arose, in part, because both systems took responsibility for primary care management although, from the patient perspective, IHS/tribe should function as the main primary care provider. Without a mechanism to regularly share medical records these sister federal agencies cannot coordinate primary care effectively.

Our findings build upon prior research on use of multiple healthcare organizations. Both VHA and IHS enrollees seek care in a combination of the private and public sector. Dual users in both the VHA and IHS tend to be older, better educated, have other public or private insurance, and have worse health compared to non-dual users. ${ }^{12,29-33}$ Of particular relevance to our study, among VHA-users, dual use has been associated with dissatisfaction in VHA healthcare, and when coupled with resources to access other options, leads to VHA services supplementing non-VHA primary care. ${ }^{11,29,32,34}$ In contrast, among IHS-users, insufficiency of IHS resources is often associated with supplementation of IHS/tribal primary care. ${ }^{12,14,17,35}$ Our focus groups confirm the finding of resource availability driving care patterns for dual users.

There was a strong preference to improve inter-organizational coordination through a shared electronic health record (EHR). These organizations cooperated in the development of their respective EHRs, which are integrated into their clinical practices. Clinicians recognized a problem in quality of care and were prepared to immediately implement improvements to benefit their patients, highlighting the presence of buy-in from the field for greater coordination of care. ${ }^{36}$ Closer alignment of these federal organizations has implications outside of health care delivery for AIAN veterans, who comprise about $1 \%$ of the US and veteran populations. By demonstrating leadership in coordination of care, VHA and IHS can demonstrate how to overcome technical, policy and administrative challenges in implementing the Institute of Medicine ${ }^{37}$ recommendations to enhance quality through data sharing and care coordination.

Issues of access and preference were raised in all stakeholder groups. Distance between VHA and IHS/tribal facilities was a concern because of cost and inconvenience, with local delivery of on-going chronic care preferred by patients and providers. We did not identify systematic differences in the VHA-IHS relationship based on distance between facilities. Consistent with prior studies, ${ }^{11}$ we found that increased distance did not diminish AIAN patients' use of VHA; perhaps due to fewer acceptable non-VHA and non-IHS options in these rural areas.

Patients were generally satisfied with the quality of care in either organization; however all stakeholders agreed that neither organization was fully culturally competent. IHS/tribes lacked competence about veterans' health needs and VHA lacked competence about AIAN patients' health beliefs and behaviors. Future educational interventions should have strong experiential components and involve local tribes to address specific cultural issues.

Focus groups are limited to interpretative insights. Although we achieved sample adequacy, the total variation in VHA-IHS relationships may not be represented, such as clusters with fewer dual users or successful joint pilot projects, including telepsychiatry. ${ }^{38}$ Since VHA catchment areas included multiple tribal communities, we cannot determine the bias of sampling at only one of these Native communities, although often members of other local tribes were invited to participate.
We acknowledge that our recruitment process may have introduced bias toward the interests of a tribal council or COS, nevertheless participants freely critiqued their respective organizations.

Our focus group research allowed the definition of a range of beliefs and elicited potential actions based on individuals' authentic experiences. Healthcare providers consistently welcomed closer collaboration between VHA and IHS to improve information flow and processes of care for mutual patients. It appears that buy-in already exists among clinicians to implement systematic changes to improve care for mutual patients in these federal organizations. Their various recommendations including a shared electronic health record and formalized regional/local referral process will require both local and national implementation strategies. Future research should evaluate local strategies to foster greater coordination as potential interventions that might be broadly adopted, while future policy initiatives should focus on sharing medical information.

Acknowledgment: This study, "VA and Indian Health Service: Access for American Indian Veterans," was funded by the VA Health Services Research and Development Service, ACC 03-304. We gratefully acknowledge the advice of the study's national VHA-IHS Advisory Committee and Elizabeth M. Yano, PhD, MSPH, of the VA Center for the Study of Healthcare Provider Behavior, for their comments and suggestions on this article. The project also benefited from technical assistance in developing the moderator discussion guide from Melissa Scherwinski, MSW of the VA Employee Education System, Steven P. Wallace, PhD of UCLA Center for Healthcare Policy and Diane Weiner, $\mathrm{PhD}$, consulting anthropologist. We also acknowledge the assistance of local site principle investigators at the participating Veterans Health Administration (VHA) and Indian Health Service (IHS) facilities, and thank the Tribal Councils for allowing us to conduct focus groups with stakeholders in their respective communities. This report presents findings and conclusions of the authors; it does not necessarily reflect the opinions or policies of the Department of Veterans Affairs or the Indian Health Service.

Conflict of Interest: None disclosed.

Corresponding Author: B. Josea Kramer, PhD; Geriatric Research Education and Clinical Center, VA Greater Los Angeles Healthcare System, 16111 Plummer Street, Sepulveda, CA 91343, USA (e-mail: Josea.Kramer@va.gov).

\section{REFERENCES}

1. US Department of Health and Human Services, Public Health Service, Indian Health Service. Collaboration between the Indian Health Service and the Veterans Health Administration. Available at: http://www.vha. ihs.gov. Accessed February 12, 2009.

2. US Department of Veterans Affairs. Federal Benefits for Veterans and Dependents (2008 Edition). Available at: http://wwwl.va.gov/opa/ vadocs/fedben.pdf. Accessed February 12, 2009.

3. US Department of Health and Human Services, Public Health Service, Indian Health Service. Frequently Asked Questions. Available at: http:// www.ihs.gov/GeneralWeb/HelpCenter/CustomerServices/FAQ/index. asp. Accessed February 12, 2009.

4. Rhoades ER. American Indian and Alaska Native health services as a system of rural health care. In: Loue S, Quill BE, eds. Handbook of rural health. New York: Kluwer Academic/Plenum Publishers; 2001:119-33.

5. Kunitz SJ. The history and politics of US health care policy for American Indian and Alaska natives. AJPH. 1996;86(10):1464-73. 
6. US Department of Health and Human Services, Public Health Service, Indian Health Service. Indian Health Manual. Available at: http://www.ihs.gov/ PublicInfo/Publications/IHSManual/index.cfm. Accessed February 12, 2009.

7. Fleming $\mathbf{C}$, Fisher ES, Chang $\mathbf{C}$, et al. Studying outcomes and hospita utilization in the elderly: the advantages of a merged data base for Medicare and Veterans Affairs hospitals. Med Care. 1992;30:377-88.

8. DeVito CA, Morgan RO, Virnig BA. Use of Veterans Affairs medical care by enrollees in Medicare HMOs. Neng J Med. 1997;337:1014-15.

9. Passman LJ, Garcia RE, Campbell L, Winter E. Elderly veterans receiving care at a Veterans Affairs Medical Center while enrolled in Medicare-Financed HMOs: Is the taxpayer paying twice? J Gen Intern Med. 1997; 12:247-49.

10. Rabiner DJ, Branch LG, Sullivan RJ. The receipt of prevention services by veterans using VA versus non-VA facilities. Prev Med. 1998;27:690-96.

11. Borowsky SJ, Cowper DC. Dual use of VA and non-VA primary care. J Gen Intern Med. 1999;14:274-80.

12. Hoff RA, Rosenheck RA. Cross-service use among psychiatric patients: Data of Veterans Affairs. J Behav Health Serv Res. 2000;27:98-106.

13. Cunningham PJ. Access to care in the Indian health service. Health Affairs. 1993;12(3):224-33.

14. Cunningham PJ, Cornelius LJ. Access to ambulatory care for American Indians and Alaska Natives: The relative importance of personal and community resources. Soc Sci Med. 1995;40:393-407.

15. Beauregard KM, Cunningham PJ, Cornelius LJ. Access to health care: Findings from the Survey of American Indians and Alaska Natives. AHCPR Pub No. 91-0028. National Medical Expenditure Survey Research Findings, 9, Agency for Health Care Policy and Research. Rockville, MD: Public Health Service; 1991.

16. Cunningham PJ, Altman BM. The use of ambulatory health care services by American Indians with disabilities. Med Care. 1993;31:606-16.

17. Shen Y, Hendricks A, Zhang S, Kazis LE. VHA enrollees' health care coverage and use of care. Med Care Rev. 2003;60:253-67.

18. Institute of Medicine. Crossing the quality chasm: A new health system of the 21 st century. Washington, DC: National Academy Press; 2001

19. Kramer, BJ, Wang M, Jouldjian S, Lee ML, Finke B, Saliba D. Healthcare for American Indian and Alaska native veterans: The roles of the veterans health administration and the Indian Health Service. Medical Care. [In press].

20. Harada ND, Villa VM, Reifel N, Bayhylle R. Exploring veteran identity and health services use among Native American veterans. Military Medicine. 2005;170(9):782-6.

21. Gurley D, Novins DK, Jones MC, et al. Comparative use of biomedical and traditional healing options by American Indian veterans. Psychiatric Services. 2001;52:68-74.

22. QSR N6 [computer program]. Edition 1. Melbourne, Australia: QSR International Pty. Ltd; March 2002.
23. Strauss A, Corbin J. Basics of qualitative research: Grounded theory procedures and techniques Newbury Park, CA: Sage; 1990.

24. Strauss A, Corbin J. Grounded theory methodology: An overview. In: Denzin NK, Lincoln YK, eds. Handbook of qualitative research. Thousand Oaks, CA: Sage; 1994:273-85.

25. Weller SC, Romney AK. Systematic data collection. Newbury Park, CA: Sage; 1988.

26. Krueger RA. Focus Groups: A practical guide to applied research. Newbury Park CA: Sage; 1988.

27. Petersen LA, Wright S. Does the VA provide "primary" primary care? J Gen Int Med. 1999;14:318-19.

28. Mitchell SM, Shortell SM. The governance and management of effective community health partnerships: A typology for research, policy, practice. Milbank Quarterly. 2000;78:241-89.

29. Stroupe KT, Hynes DM, Giobbe-Hurder A, Oddone EZ, Weinberger M, Reda DJ, Henderson WG. Patient satisfaction and use of Veterans Affairs versus non-Veterans Affairs healthcare services by veterans. Med Care. 2005;43:453-60.

30. Harada ND, Villa VM, Andersen R. Satisfaction with VA and Non-VA outpatient care among veterans. Am J Med Qual. 2002;17(4):158-64.

31. Agha Z, Lofgren RP, VanRuiswyk JV, Layde PM. Are patients at Veterans Affairs medical centers sicker?: A comparative analysis of health status and medical resource use. Arch Intern Med. 2000;160: 3252-57.

32. Ross JS, Keyhani S, Keenan PS, Bernheim SM, et al. Use of recommended ambulatory care services: Is the Veterans Affairs quality gap narrowing? Arch Intern Med. 2008;168(9):950-58.

33. Bean-Mayberry B, Chang C, McNeil M, Hayes P, Scholle SH. Comprehensive care for women veterans: Indicators of dual use of VA and NonVA Providers. J Am Med Women's Assoc. 2004;59(3):192-97.

34. Hynes DM, Koelling K, Stroupe K, Arnold N, Mallin K, Sohn MW, Weaver FM, Manheim L, Kok L. Veterans' access to and use of Medicare and Veterans Affairs health care. Med Care. 2007;45(3):214-23.

35. Baldwin L, Hollow WB, Casey S, Hart LG, Larson EH, Moore K, Lewis E, Andrilla CHA, Grossman DC. Access to specialty health care for rural American Indians in two states. J Rural Health. 2008;24(3):269-78.

36. Rubenstein LV, Mittman BS, Yano EM, Mulrow CD. From understanding health care provider behavior to improving health care. Medical care 2000;38 [supple I-129 - I-141.

37. Institute of Medicine, Committee on Enhancing Healthcare Quality Programs. Leadership by example: Coordinating government roles in improving health care quality. In: Corrigan JM, Smith BM, eds. Washington DC: National Academy Press; 2002

38. Shore JH, Savin D, Orton H, Beals J, Manson SM. Diagnostic reliability of telepsychiatry in American Indian veterans. Am J Psychiatry. $2007 ; 164: 115-18$ 